\title{
Analyzing the Performance of Coded OFDM based WiMax System with different Fading Conditions
}

\author{
Hardeep Kaur and M. L Singh \\ Department of Electronics Technology, Guru Nanak Dev University, Amritsar, India \\ hardeepgndu@gmail.com,mlsingh7@gmail.com
}

\begin{abstract}
The demands for high speed applications are growing at a faster rate. To meet theserequirements, $4 G$ technologies are being used nowadays. COFDM based WiMax is the outcome in this direction which promises to cater these high speed and high quality applications. Worldwide Interoperability for Microwave Access (WiMax) is an IEEE 802.16 standard-based broadband wireless access (BWA) technology which employsCoded orthogonal frequency division multiplexing access (COFDM). This paper analyses Bit Error Rate for WiMax based COFDM system with QPSK modulation scheme under various channel conditions like AWGN, Rayleigh, Rician and Nakagami-m. It has been observed that performance of Nakagami fading channel is better than other fading channels.
\end{abstract}

Keywords: Worldwide Interoperability for Microwave Access, Broadband Wireless Access, Coded Orthogonal Frequency Division Multiplexing, Bit Error Rate, Nakagami

\section{Introduction}

Nowadays, the demand for wireless internet for telephony, radio and television has increased many folds. To meet these requirements and to ensure high quality of service to users, the IEEE 802.16 working group came up with a new technology called WiMax which is basically a BWA technique. WiMax is one of the promising and hottest broadband wireless access techniques being used today. It is a 4G technologybased on IEEE 801.16 specifications which promises to provide high speed and wide area coverage for both LOS (Line of Sight) and NLOS (Non Light of Sight) communication. Initially, IEEE 802.16 working group developed an air-interface standard with the aim to provide point-to-multipoint broadband services in the frequency band that spreads from 10 to $66 \mathrm{GHz}$. This standard was released in 2001. Later on, new modified standards like IEEE 802.16a and IEEE 802.16b were originated [1]. These standards addressed spectrum issues and were used at frequencies below $11 \mathrm{GHz}$. IEEE 802.16c standard provides greater interoperability in the frequency band of 10 to 66 GHz. In 2004, a new standard known as IEEE 802.16d was developed to operate in non lineof-sight (NLOS) scenario to provide fixed wireless services in 2 to $11 \mathrm{GHz}$ frequency range. This standard is based on multi carrier modulation (MCM) scheme with 256 subcarriers [2]. In December 2005, IEEE group approved a new standard known as IEEE802.16e, which is an amendment to IEEE 802.16d standard that supports mobility services as well.

This standard also supports scalable Orthogonal Frequency Division Multiple Access (SOFDMA) which enables operation over several bandwidths ranging from 1.5 to $20 \mathrm{MHz}$. TheIEEE 802.16e standard is often referred as Mobile WiMax [3]. Currently the IEEE 802.16-2009 is developed which is extension of IEEE 802.16j. The IEEE 802.16j presents amendments in IEEE $802.16 \mathrm{e}$ standard by incorporating relay-based multi hop network 
capability [4]. These standards are for Mobile WiMax but focus of present work will be on Fixed Mobile. Fixed WiMAX incorporates COFDM as MCM technique.

In recent years, orthogonal frequency-division multiplexing (OFDM) has emerged as the standard of choice in a number of important high-data-rate applications. In OFDM, instead of using a single wide-band carrier to transmit information, a large number of parallel narrowband sub-carriers are used. In OFDM serial-to-parallel transmitter converts the incoming high-rate data stream into low-rate streams, and then transmits each low-rate data stream over a unique orthogonal carrier. The data rate of each transmitted stream is effectively reduced by a factor of $\mathrm{N}$ from the original data rate. Utilizing this strategy, OFDM drastically reduces inter symbol interference (ISI) by avoiding multipath in frequency-selective channels. In 2011, performance of COFDM was analyzed for various fading environments for WLAN standard [5].

In this paper, a model of an OFDM system employing convolutional coding for WiMaxis developed using MATLAB for various channels namely AWGN, Rayleigh, Rician and Nakagami-m using QPSK. The main objective of the work is to analyse the performance of system for various fading environments for WiMax.

\section{IEEE 802.16d Specifications}

IEEE 802.16 is a set of standards used for providing wireless multimedia services in Wireless Metropolitan Areas. The IEEE 802.16d standard came into existence in 2004 which operates on both 10-66 GHz (LOS) and 2-11 GHz (NLOS) frequency range. All the previous standards were limited for line-of-sight (LOS) communication. TheIEEE 802.16d provides NLOS propagation by making use of OFDM and OFDMA.The Line-of-sight propagation can be carried by making use of single carrier modulation. This standard specifies an OFDM physical layer that splits an information signal across 192 separatedata subcarriers. The resulting subcarrier frequency spacing is $31.25 \mathrm{KHz}$ with 256 frequency slots. Table1 shows all the parameters considered for carrying out simulation work based on this standard. The total symbol duration is $40 \mu \mathrm{s}$ with a cyclic prefix ratio of 0.25 . The total symbol duration changes with the change in cyclic prefix ratios.

Table 1. Simulation Parameters

\begin{tabular}{|l|l|}
\hline Parameter & Value \\
\hline No. of data subcarriers & 192 \\
\hline FFT Size & 256 \\
\hline No. of OFDM Symbols & 10000 \\
\hline FFT sampling frequency & $8 \mathrm{MHz}$ \\
\hline Cyclic prefix Interval & $1 / 4,1 / 8,1 / 16$ \\
\hline Data symbol duration & $32 \mu \mathrm{s}$ \\
\hline Cyclic prefix duration & $8 \mu \mathrm{s}, 4 \mu \mathrm{s}$ and $2 \mu \mathrm{s}$ \\
\hline Total symbol duration & $40 \mu \mathrm{s}, 36 \mu \mathrm{s}$ and $34 \mu \mathrm{s}$ \\
\hline Channel Coding & $\begin{array}{l}\text { Convolutional Coding with data rate of } 1 / 2,2 / 3, \\
3 / 4 \text { and } 5 / 6\end{array}$ \\
\hline Modulation Scheme & QPSK \\
\hline Channel conditions & AWGN, Rayleigh, Rician, Nakagami-m \\
\hline
\end{tabular}




\section{Implementation of OFDM}

To implement the OFDM transmission scheme, the whole design is divided into three sections: Transmitter, Channel and Receiver as shown in Figure 1.

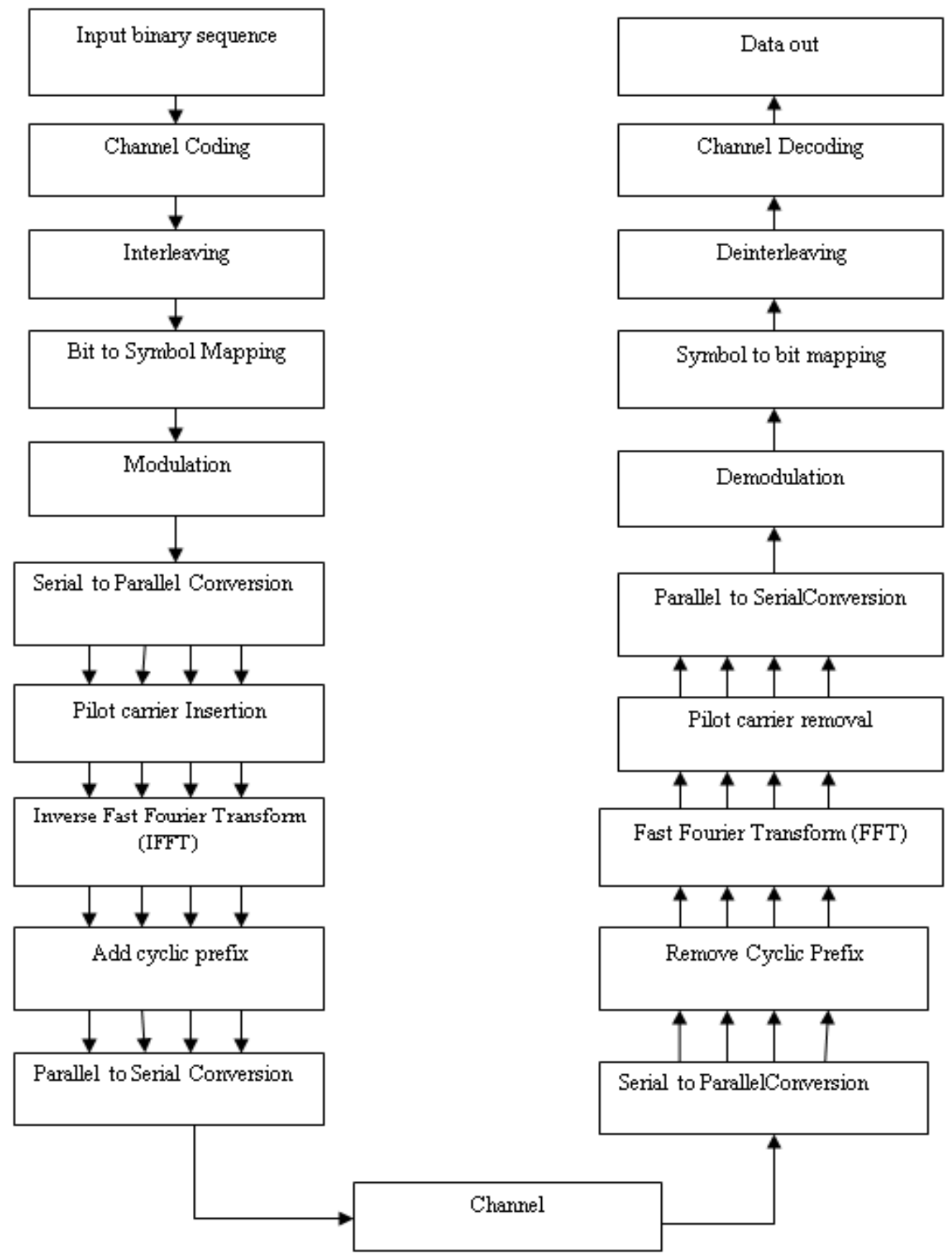

Figure 1. Block Diagram of OFDM System

In the transmitter, random binary input data sequence is generated. Channel Coding can be provided using Forward Error-Correction Coding (FEC) like block code and convolutional 
code $[6,7]$. Further, interleaving is done to provide frequency diversity [8]. Thus the input sequence is encoded by a FEC encodercomprising of convolutional codes. Then Interleaving is applied to randomize the occurrence of bit errors to increase performance. After interleaving, the binary values are converted to symbol values, on which modulation scheme BPSK is carried out. The modulated data is then divided into low data rate streams using serial to parallel conversion. Each parallel stream is then modulated on a separate subcarrier in the allocated spectrum using IFFT. Previously, multi-carrier systems were implemented using of separate local oscillators which was both inefficient and costly. But with the advent of cheap powerful DSP processors, the sub-carriers can now be implemented by the FFT which keep subcarriers to orthogonal with each other. The symbol is modulated onto sub carriers by applying the Inverse Fast Fourier Transform (IFFT). Then cyclic extension is added to make the system robust to multipath propagation. The parallel data is then further converted to serial form using serial to parallel conversion. For WiMax system, 200 subcarriers (192 data and 8 pilotsubcarriers) are used while 56 null subcarriers are used thus making FFT size equal to 256.This serial data is then transmitted over multipath fading channel. The channel can be additive white Gaussian noise (AWGN). The multipath fading channels used for this work are Rayleigh, Rician and Nakagami-m. The receiver performs the reverse operations of the transmitter. After removing the cyclic extension, the signal can be applied to a Fast Fourier Transform (FFT) to recover the modulated values of all subcarriers. The modulated values are then demapped into binary values, and finally deinter leaving and decoding is performed to get back information bits.

\section{Fading Conditions}

Multipath fading is a very common process in wireless communication system. In this paper, the following fading channels have been used in addition to AWGN channel.

\subsection{Rayleigh Fading}

Rayleigh fading is applicable for non-line of sight (NLOS) propagation. The signal received at the receiver will be combination of reflected and scattered replicas of transmitted signal. The received signal $\mathrm{s}(\mathrm{t})$ can be expressed as

$s(t)=\sum_{i=1}^{N} a_{i} \cos \left(w_{c} t+\varphi_{i}\right)$

where $N$ is number of replica paths, $w_{c}$ is carrier frequency and $\varphi_{i}$ is phase of signal on $i$ th path.

If there is a relative motion between transmitter and receiver the Doppler shift should be considered. The received signal $s(t)$ under this condition can be expressed as

$s(t)=\sum_{i=1}^{N} a_{i} \cos \left(w_{c} t+w_{d i} t+\varphi_{i}\right)$

where $w_{d i}$ is Doppler shift for $i$ th path.

The phase $\varphi_{i}$ is assumed to be uniformly distributedover $(0,2 \pi)$. If $\mathrm{N}$ is large, the in-phase and quadrature components of received signal becomes zero mean Gaussian with standard deviation $\sigma$. The probability density function (PDF) of the received signal envelope can be given as $[5,9,10]$

$f(r)=\frac{r}{\sigma^{2}}\left\{\frac{-r^{2}}{2 \sigma^{2}}\right\}, \quad r \geq 0$ 


\subsection{Rician Fading}

Rician fading is similar to Rayleigh fading but it considers LOS propagation instead of NLOS as in the case of Rayleigh fading. In this scenario, if $k_{d}$ is the strength of LOS component and $w_{d}$ is the Doppler shift along the LOS path, the received signal $s(t)$ can be written as $[10,11,14]$

$s(t)=\sum_{i=1}^{N} a_{i} \cos \left(w_{c} t+w_{d i} t+\varphi_{i}\right)+k_{d} \cos \left(w_{c} t+w_{d} t\right)$

The probability density function (PDF) of the received signal envelope can be given as

$f(r)=\frac{r}{\sigma^{2}}\left\{-\frac{r^{2}+k_{d}^{2}}{2 \sigma^{2}}\right\} I_{0}\left\{\frac{r k_{d}}{\sigma^{2}}\right\}, \quad r \geq 0$

\subsection{Nakagami-m Fading}

The Nakagami distribution is also known as m-distribution. This distribution gives better result than Rayleigh and Rician distributions. The PDF of the received signal envelope for this type of distribution is given by

$f(r)=\frac{r}{\Gamma(m)}\left(\frac{m}{\Omega}\right)^{m} r^{2 m-1} e^{-m r^{2} / \Omega}, \quad r \geq 0$

where $m$ is shape factor, $\Omega$ is second moment of $r$ and $\Gamma($.$) is gamma function. The parameter$ $\Omega$ controls the spread of distribution. The value of $\mathrm{m}$ determines the type of distribution. When $m=1$ the distribution becomes Rayleigh distribution. For $m \geq 1$ the fading effect becomes less severe. When $\mathrm{m}$ is in between 1 and 2 , then the distribution becomes Rician. Therefore Nakagami distribution encompasses both the distributions. When $m=\infty$, the effect of fading totally diminishes [11].

\section{Simulation Results}

This section analyzed the performance of WiMax system using QPSK modulation for different channels. The BER for these channels is investigated and the results are plotted.

\subsection{WiMax Under Different Fading Channel Conditions for QPSK}

Under this section, the comparison of BER under various channel conditions is done. Convolutional code of code rate $1 / 2$ is used. The number data subscribers used is 192 with FFT size of 256. The value of guard interval is $1 / 4$. The value of $m$ is chosen as 3 for Nakagami-m distribution.

Table 2. BER for AWGN, Rayleigh, Rician and Nakagami-m Channels

\begin{tabular}{|c|c|c|c|c|}
\hline Eb/N0 & AWGN & Rayleigh & Rician & Nakagami-m \\
\hline 0 & 0.2747 & 0.4964 & 0.4799 & 0.4576 \\
\hline 2 & 0.0567 & 0.4834 & 0.4166 & 0.3498 \\
\hline 4 & 0.0011 & 0.4443 & 0.2535 & 0.1524 \\
\hline 6 & 0.0000 & 0.3382 & 0.0627 & 0.0204 \\
\hline 8 & 0 & 0.1800 & 0.0047 & 0.0015 \\
\hline 10 & 0 & 0.0565 & 0.0002 & 0.0001 \\
\hline 12 & 0 & 0.0098 & 0.0000 & 0.0000 \\
\hline
\end{tabular}




\begin{tabular}{|c|c|c|c|c|}
\hline 14 & 0 & 0.0012 & 0.0000 & 0 \\
\hline 16 & 0 & 0.0001 & 0 & 0 \\
\hline 18 & 0 & 0.0000 & 0 & 0 \\
\hline 20 & 0 & 0 & 0 & 0 \\
\hline
\end{tabular}

Figure 2 shows the BER comparison of the COFDM based WiMax system under various fading environments. It is evaluated that BER for AWGN is less than other channels.

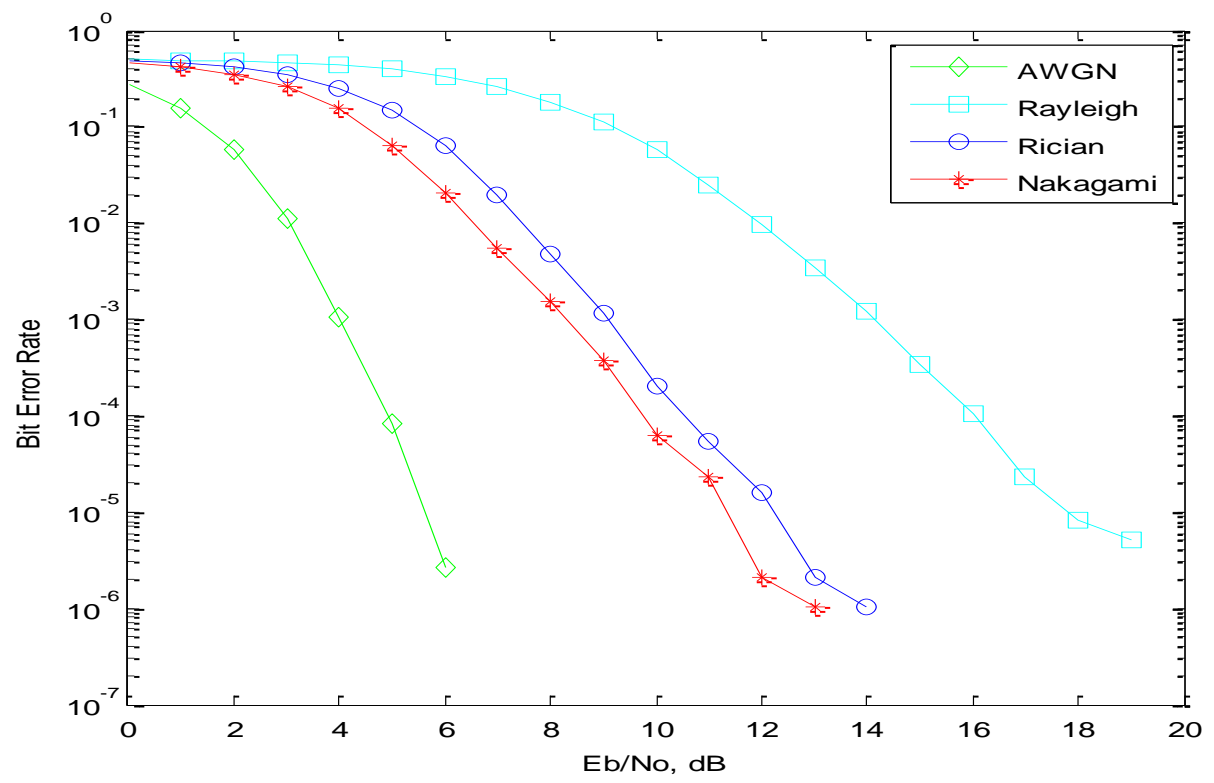

Figure 2. WiMax under Various Channel Conditions like AWGN, Rayleigh, Rician, Nakagami-m for QPSK with Guard Interval of 0.25

It is observed that theBER for AWGN is less than other channels for a particular Eb/N0 as shown in Table 2. For Eb/N0 equal to $4 \mathrm{~dB}$, the BER for AWGN, Rayleigh, Rician and Nakagami-m is $0.0011,0.4443,0.2535$ and 0.1524 respectively. It is also observed that Nakagami-m give better performance than Rayleigh and Rician fading channel. Similar types of trends can also be observed for WLAN systems [11].

\subsection{WiMax for AWGN Channel with Different Code Rates}

In this we investigation of the AWGN channel for WiMax system employing convolutional coding with QPSK modulation is done. Figure 3 shows the behavior of System for AWGN channel for different code rates with guard interval of 0.25 . The code rates are taken as 1/2, 2/3, 3/4 and 5/6..For Eb/N0 equal to $4 \mathrm{~dB}$, the BER for code rate of 1/2, 2/3, 3/4 and $5 / 6$ is $0.0002,0.0320,0.0977$ and 0.2114 respectively. It is observed that as code rate increases, the BER decreases [12-14]. 


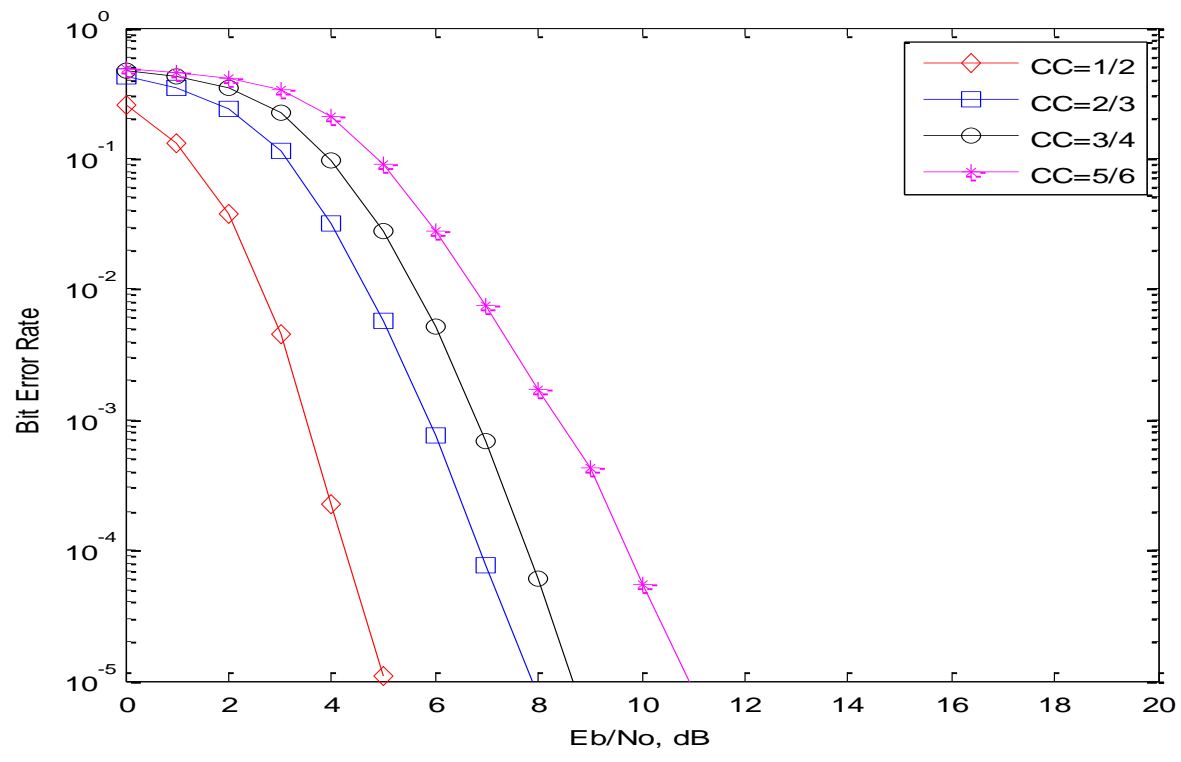

Figure 3. WiMax for AWGN Channel with Different Code Rates for QPSK

\subsection{WiMax for Rayleigh Fading Channel with Different Code Rates}

In this investigation of the Rayleigh channel for WiMax system employing convolutional coding with QPSK modulation is done. Figure 4 shows the behavior of System for Rayleigh channel for different code rates with guard interval of 0.25 . The code rates are taken as $1 / 2$, $2 / 3,3 / 4$ and 5/6..For Eb/N0 equal to $4 \mathrm{~dB}$, the BER for code rate of 1/2, 2/3, 3/4 and 5/6 is $0.4354,0.4860,0.4943$ and 0.4950 respectively. It is observed that as code rate increases, the BER decreases.

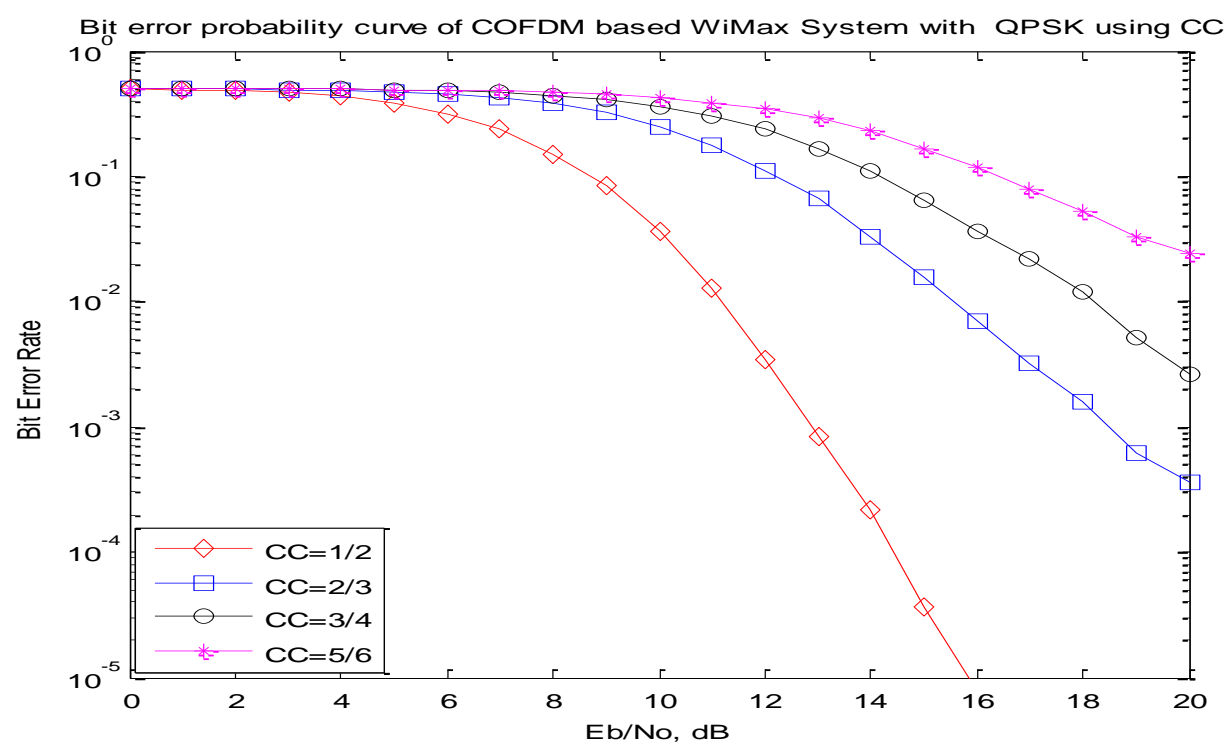

Figure 4. WiMax for Rayleigh Channel with Different Code Rates for QPSK 


\subsection{WiMax for Rician Fading Channel with Different Code Rates}

In this investigation of the Rician channel for WiMax system employing convolutional coding with QPSK modulation is done. Figure 5 shows the behavior of System for Rician channel for different code rates with guard interval of 0.25 . The code rates are taken as $1 / 2$, $2 / 3,3 / 4$ and 5/6..For Eb/N0 equal to $4 \mathrm{~dB}$, the BER for code rate of $1 / 2,2 / 3,3 / 4$ and 5/6 is $0.2295,0.4176,0.4630$ and 0.4794 respectively. It is observed that as code rate increases, the BER decreases.

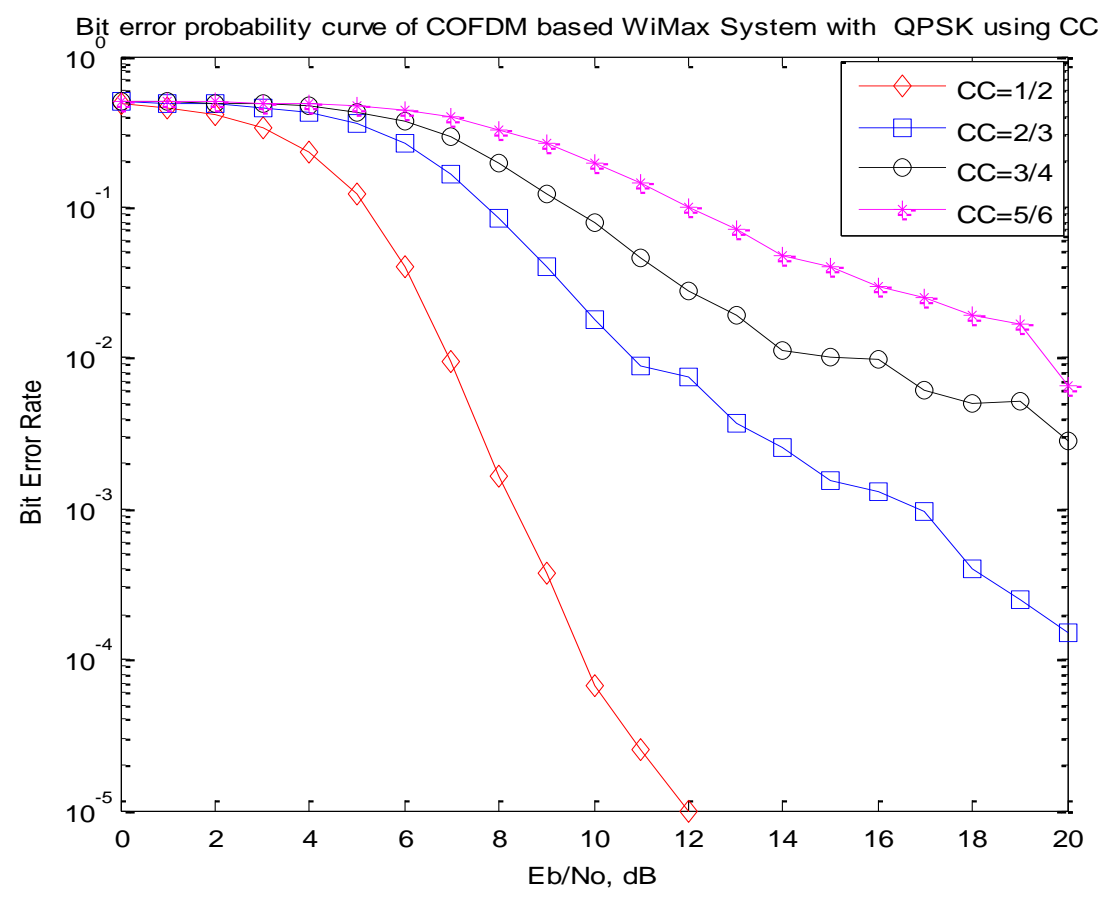

Figure 5. WiMax for Rician Channel with Different Code Rates for QPSK

\subsection{WiMax for Nakagami-m Fading Channel with Different Code Rates}

In this investigation of the Nakagami-m channel for WiMax system employing convolutional coding with QPSK modulation is done. Figure 6 shows the behavior of System for Nakagami-m channel for different code rates with guard interval of 0.25 . The code rates are taken as 1/2, 2/3, 3/4 and 5/6..For Eb/N0 equal to $4 \mathrm{~dB}$, the BER for code rate of 1/2, 2/3, $3 / 4$ and $5 / 6$ is $0.1233,0.3566,0.4293$ and 0.4631 respectively. It is observed that as code rate increases, the BER decreases. 


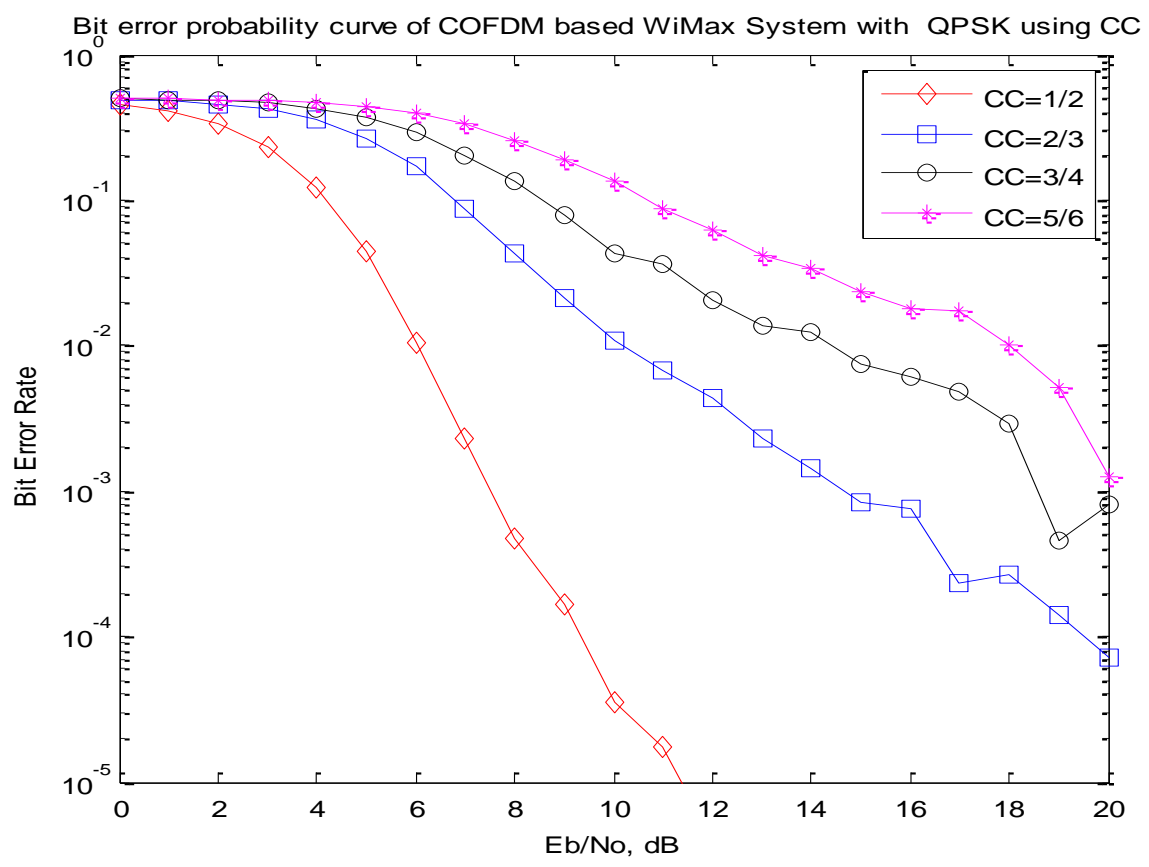

\section{Figure 6. WiMax for Nakagami-m Channel with Different Code Rates using QPSK}

\section{Conclusion}

In this paper the performance of COFDM based physical layer of WiMax system is investigated under various fading channels using QPSK. The three fading channels namely Rayleigh, Rician and Nakagami-m are examined using convolutional coding. It is concluded that performance of AWGN channel is best among all channels while Rayleigh fading channel gives worst performance in terms of BER. The performance of Nakagami-m channel is better than Rayleigh and Rician. Further the work is carried out for Rayleigh, Ricianand Nakagami-m channels with different code rates.It is concluded that convolutional code of code rate $1 / 2$ gives better performance than $2 / 3,3 / 4$ and $5 / 6$ for all channels. It is also observed that as signal to noise ratio increases the BER decreases.

\section{References}

[1] B. Kamali, R.A. Bennett and D.C. Cox, "Understanding WiMax: An IEEE 802.16 Standard-basedWireless Technology”, IEEE Potentials, (2012), pp. 23-27.

[2] IEEE Standard 802.16 TM-2004, Part 16: Air interface for fixed broadband wireless access systems, (2004) October.

[3] IEEE Standard 802.16e TM-2005, Part 16: Air interface for fixed and mobile broadband wireless access systems, (2006) February.

[4] IEEE Standard 802.16 TM-2009, Part 16: Air interface for fixed and broadband wireless access systems, (2009) May.

[5] A. Joshi and D.S. Saini, "Performance Analysis of Coded-OFDM with RS-CC and Turbo codes in various fading environment", Proc. IEEE $5^{\mathrm{TH}}$ International Conference on IT and Multimedia, (2011), Malaysia.

[6] S. Bansal and R. Upadhyay, "Performance Improvement of WiMax IEEE 802.16e in Presence of different FEC Codes", Proc. IEEE International Conference on ComputationalIntelligence, Communication Systems and Networks, (2009), pp. 226-229.

[7] S. Nanda and K. M. Rege, "Frame error rates of convolutional codeson fading channels and the concept of effective Eb/N0", IEEE Trans. Veh. Tech., vol. 47, no. 1, issue 4, (1998), pp. 1245-1250. 
[8] K. Witrisal, Yong-Ho Kim and R. Prasad, "A novel approach for performance evaluation of OFDM with error correction coding and interleaving”, Proc. IEEE Veh. Technol. Conference, (1999), pp. 294-299.

[9] M. DongLin, L. Fansheng and S. Jie, "Simulation on multi-path fading in wireless channel", Proc. IEEE International Conference on Computer Science and Electronics Engineering, (2012), pp. 427-429.

[10] G. S. Prabhu and P.M. Shankar, "Simulation of flat fading using MATLAB for classroom instruction", IEEE Transactions on Education, vol. 45, issue 1, (2002), pp. 19-25.

[11] A. Joshi and D.S. Saini, "Coded-OFDM in various fading environment", Proc. IEEE International Conference, Singapore, (2010), pp. 127- 131.

[12] J.G. Proakis, "Digital Communications, McGraw Hill”, (2001).

[13] H. Kaur and M. L. Singh, "Bit Error Rate Evaluation of IEEE 802.16 (WiMax) in OFDM System", International Journal of Computer Applications, vol. 40, issue 12, (2012), pp. 10-13.

[14] M. A. Islam, R. U. Mondal and M. Z. Hasan, "Performance Evaluation of WiMAX Physical Layer under Adaptive Modulation Techniques and Communication Channels", International Journal of Computer Science and Information Security, vol. 5, issue 1, (2009), pp. 111-114.

\section{Authors}

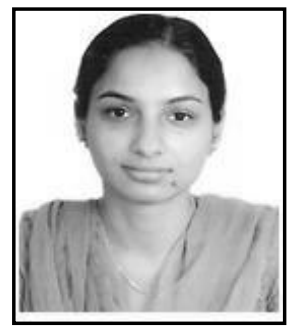

Hardeep Kaur, she received her B. Tech degree in Electronics and Communication Engineering in 2003 from Guru Nanak Dev Engineering College, Ludhiana and $\mathrm{M}$. Tech degree in same stream from Punjab Technical University, Jalandhar in 2007. She joined Guru Nanak Dev University Amritsar as Assistant Professor in the department of Electronics Technology in the year 2007. She is currently pursuing Ph. D on WiMax Networks from Guru Nanak Dev University, Amritsar. Her interests include wireless communications, digital signal processing and digital circuits.

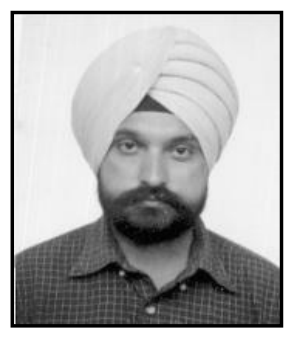

M. L Singh, he received his B. Tech degree in Electronics Engineering from Guru Nanak Dev University, Amritsar in 1991, M. Tech degree in Communication System from IIT Kanpur in 1997 and $\mathrm{Ph}$. D in Optical Communication from Guru Nanak Dev University Amritsar in 1991. He joined department of Electronics Technology as Lecturer, Guru Nanak Dev University, Amritsar in 2002.He worked as Head of Department from 2007-2010.Presently, he is working as Professor in department of Electronics Technology in the same institution. He has 75 publications to his credit in various journals and international conferences. His area of interest includes optical and wireless communications. 